amidobenzylpenicillin prepared from the $p$-aminobenzylpenicillin showed them to be indistinguishable on paper chromatograms and in respect of their antibacterial spectra.

The $p$-aminobenzylpenicillin has been converted into a series of $\mathrm{N}$-derivatives ${ }^{6}$ by treatment with reactive halogen compounds such as carboxylic acid halides, $2: 4$-dinitrofluorobenzene and $p$-toluenesulphonyl chloride, following the method of Newton and Abraham ${ }^{7}$ for the preparation of derivatives of cephalosporin $\mathrm{N}$. It is hoped to publish further details of these experiments, together with the results of the antibacterial activity of the derivatives, elsewhere, at a later date.

$$
\begin{aligned}
& \text { A. Ballio } \\
& \text { E. B. Chat } \\
& \text { F. Dentioe di Accadia }
\end{aligned}
$$

International Centre for Chemical Microbiology, Istituto Superiore di Sanita,

$$
\text { Rome. }
$$

G. N. Rolinson

F. R. BATCHELOR

Beecham Research Laboratories Limited, Brockham Park, Betchworth, Surrey.

Dec. 4.

${ }^{1}$ Corse, J. W., Jones, R. G., Soper, Q. 1'., Whitehead, C. W., and Behrens, O. K., J. Amer. Chem. Soc., 70, 2937 (1948)

a Brewer, G. A., and Johnson, M. J., App. Microbiol., 1, 163 (1953).

a Tosoni, A. L., Glass, D. G., and Goldsmith, L., Biochem. J., 69, 476 (1958).

${ }^{4}$ Abraham, E. P., Newton, G. G. F., and Hale, C. W., Biochem. J., 68, 94 (1954).

${ }^{5}$ Ford, J. H., Indust. Eng. Chem., Anal. edit., 19, 1004 (1947).

${ }^{8}$ Doyle, F. P., and Nayler, J. H. C., British Patent App. No. 13738 (1957).

'Newton, G. G. F., and Abraham, E. P., Biochem. J., 58, 103 (1954).

\section{Nucleotide Metabolism in Cardiac Activity}

Is a previous study on the nucleotide metabolism of cardiac muscle $\theta^{1}$, the changes of adenosine triphosphate were investigated during systole and diastole. Using the Cohn and Carter method of analysis' on a 'DowexI' column, the nucleotides were separated and determined quantitatively by ultra-violet absorption spectrophotometry. During the studies on the extracts of hearts frozen in systole by an acetone-dry ice mixture, a substance appered as a new peak in the chromatogram, which seemed to be an adenine nucleotide with more nitrogens than could be accounted for. This was called nucleotide $H$.

In spite of the recent results obtained by Mommaerts ${ }^{3}$ and Fleckenstein et $a l{ }^{4}$ it was decided to reinvestigate this problem, with special emphasis on nucleotide $H$.

Hearts of dogs were excised rapidly and dropped either into cold isotonic potassium chloride to stop them in diastole, or into liquid air to freeze them in systole. The hearts were then rapidly homogenized in a Waring blendor with 2.5 vol. $0.5 \mathrm{~N}$ perchloric acid, centrifuged, and the supernate neutralized to $p \mathrm{H} 8$ with potassium hydroxide. In all these steps, the temperature was kept below $5^{\circ} \mathrm{C}$. After removing the potassium perchlorate by filtration, an aliquot corresponding to about $4 \mathrm{gm}$. of the original muscle was placed on a 'Dowex-1' column in the chloride cycle, with a bed volume of either 5 or $7 \mathrm{ml}$. Elution. was carried out according to Cohn and Carter ${ }^{2}$ except that $0.005 \mathrm{~N}$, instead of $0.003 \mathrm{~N}$, hydrochloric acid was used, and this was used as the first eluant.

With this system, the results previously obtained ${ }^{x}$ were verified. With $0.005 \mathrm{~N}$ hydrochloric as eluant, adenine was eluted at $2-4$ bed volumes and inosinic acid at 15-17 bed volumes. A long trailing peak was eluted at about 50-100 bed volumes, and this was identified as adenosine diphosphate. Usually, elution was stopped after 40 bed volumes of $0.005 \mathrm{~N}$ hydrochloric acid and the next eluant started. In diastolic hearts no further peaks were obtained with the first eluant; but in systolic hearts another peak came off the column at $22-27$ bed volumes, which corresponded to the previously identified nucleotide $H$. This fraction was collected, neutralized to $p \mathrm{H} 7$, and concentrated in vacuo at a temperature below $50^{\circ} \mathrm{C}$. On phosphorus analysis ${ }^{5}$, one organic and usually one inorganic phosphorus were obtained per mole adenine, determined spectrophotometrically. Occasionally more than one inorganic phosphorus was obtained. On paper chromatography, using Hanes and Isherwood's ${ }^{8}$ method to determine the phosphorus spots, and Magasanik's? method to determine the purine spots, a phosphorus spot was always associated with the purine spot, at an $R_{F}$ value that corresponded to adenylic acid. On mixing some of the unknown with authentic adenylic acid, the purine spots could not be separated on paper.

Furthermore, on paper chromatography there were always two ninhydrin spots identified as aspartic and glutamic acids, with occasionally a third one identified as alanine. Again, on mixing the unknown with authentic glutamic and aspartic acids, no separation occurred on paper.

On rechromatography on 'Dowex-1', or following the precipitation of the nucleotides with mercuric acetate $^{8}$, the two amino-acids and the adenylie acid spots were always present, and yet they never chromatographed as one unit on paper. Using the solvent system, isobutyric acid/ammonia/water, $66: 1: 33$, the $R_{F}$ value for nucleotide $H$ was 0.51 , adenylic acid $0 \cdot 51$, glutamic acid $0 \cdot 45$, and aspartic acid $0 \cdot 35$. A mixture of adenylic, aspartic and glutamic acids acted in all respects like nucleotide $H$.

Thus, in conclusion, the previously named nucleotide $H$ is only an artefact made up of adenylic, aspartic and glutamic acids. On the anion exchange resin, 'Dowex-1', it happened that these three compounds are eluted from the column at the same rate, and are precipitated together by mercuric acetate.

The reason why the two amino-acids appear only in systolic hearts remains to be investigated.

Philip A. Khairallah*

Department of Biochemistry,

American University of Beirut, Beirut.

Oct. 23.

* Present address : Cleveland Clinic, 2020 East 93rd Street, Cleveland 6, Ohio. Research Fellow, American Heart Association.

${ }^{1}$ Khairallah, P. A., and Mommaerts, W. F. H. M., Circul. Res., 1, 12 (1954).

${ }^{2}$ Cohn, W. E., and Carter, C. F., J. Amer. Chem. Soc., 72, 4273 (1950)

${ }^{3}$ Mommaerts, W. F. H. M., Amer. J. Physiol., 182, 585 (1955).

4 Fleckenstein, A., Janke, J., Davies, R. E., and Krebs, H. A., Nature, 174, 1081 (1954).

${ }^{5}$ Fiske, C. H., and Subbarow, Y., J. Biol. Chem., 66, 375 (1925).

'Hanes, C. S., and Isherwood, F. A., Nature, 164, 1107 (1949).

' Magasanik, B., Vischer, E., Doniger, R., Elson, D., and Chargaff, E., J. Biol.' Chem., 186, 39 (1950).

${ }^{8}$ Kerr, S. E., J. Biol. Chem., 145, 647 (1942). 